\title{
Trend of asthma prevalence among children based on regional urbanization level in Japan; 2006-2019
}

\author{
Tasuku Okui * (D) \\ Medical Information Center, Kyusyu University Hospital, 3-1-1, Maidashi Higashi-ku, Fukuoka city 812-8582, Japan \\ *Correspondence: task10300@gmail.com
}

Received: October 8, 2021 Accepted: November 20, 2021

\begin{abstract}
Although it has been known that the prevalence of asthma tends to be higher among children in the metropolitan areas of Japan, trends of the prevalence with respect to the regional urbanization level has not been investigated in recent years. We investigated trends in the prevalence of asthma among children and air pollutant concentrations by regional urbanization levels using data from the School Health Statistics Survey in Japan from 2006 to 2019. We calculated the age-standardized prevalence of asthma for each year, gender, regional urbanization level, and annual percent change (APC). In addition, the slope index of inequality (SII) and relative index of inequality (RII) were calculated for evaluating disparity in age-standardized asthma prevalence depending on regional urbanization levels. Moreover, we calculated the mean of the annual average values by regional urbanization levels for sulfur dioxide $\left(\mathrm{SO}_{2}\right)$, nitrogen dioxide $\left(\mathrm{NO}_{2}\right)$, suspended particulate matter (SPM), carbon monoxide (CO), and photochemical oxidant (Ox) from 2006 to 2018 . We found that the age-standardized prevalence significantly decreased in the periods in the metropolis for males and females, and the degree of the decrease was largest in the metropolis. Conversely, the age-standardized prevalence increased in towns and villages, and the APC was greater than zero. In addition, both the SII and RII showed significant decreasing trends in the study period, and the regional disparity shrank over the years. Moreover, concentrations of the air pollutants were highest in the metropolis throughout the years except for Ox, whereas the difference in the concentrations of $\mathrm{NO}_{2}$, SPM, and $\mathrm{CO}$ decreased between the metropolis and the other areas over the years. In conclusion, disparity in asthma prevalence depending on regional urbanization level decreased from 2006 to 2019, and there is a possibility that regional difference in trend of the air pollutants is related to the result.
\end{abstract}

Keywords: children, asthma, Japan, prevalence, air pollution

\section{Introduction}

Asthma is one of the common diseases with a high prevalence in children. Globally, the prevalence of asthma varies per region, and a high prevalence is observed in resource-rich countries [1]. In addition, it is known that the prevalence of asthma particularly increased in children over the last 40 years [2]. In Japan, number of patients with asthma has been fluctuating between 1984 and 2014, whereas the mortality rate has been on a continuous decrease over the years [3]. The prevalence of asthma is particularly high in children [3] and investigating the causes and preventive measures for asthma in children is of prime importance in Japan.

The prevalence of asthma varies with respect to region also in Japan, and urban areas have often been shown to be associated with a higher prevalence of asthma [4,5]. A previous study in Japan analyzed the School Health Statistics Survey in 2006 and revealed that the prevalence of asthma tended to be higher among children in metropolitan areas compared with the other regions [6]. Conversely, the difference in the prevalence has not been investigated, and it is unknown whether the difference increased or decreased over the years. In addition, air pollution is a major risk factor for asthma [7], and an increase in air pollutant concentrations following the economic development after World War II has caused a major pollution problem, which led to a disease called Yokkaichi asthma in Japan [8]. However, differences in trends of air pollutant concentrations by urbanization levels of various regions have not been investigated in Japan in recent years. Air pollutant concentrations decreased in Tokyo over the recent decades [9,10], and the relationship between regional urbanization levels and the prevalence of asthma may have changed in recent years. Moreover, investigating the trend of air pollutant concentrations by regional urbanization level will be meaningful for understanding the trends in the prevalence of asthma in Japan.

We investigated the trends in the prevalence of asthma and air pollutant concentrations among children by regional urbanization level using nationwide government statistics of Japan. 


\section{Materials and Methods \\ Data}

Data from the School Health Statistics Survey conducted by the ministry of education, culture, sports, and science in Japan was used [11]. The School Health Statistics Survey aims at investigating the development and health status of children in schools. The survey is conducted every year, and randomly chosen schools in Japan respond to the survey. In the survey, schools are stratified by number of children for each prefecture and type of school, and target schools are randomly chosen from each stratum in each prefecture [12]. Over 3 million children become targets of the health status survey every year [12]. Data on the prevalence of asthma by urbanization level for children aged 6-17 years from 2006 to 2019 were used in this study. Urbanization levels consist of the metropolises, medium sized cities, small sized cities, and towns and villages. The metropolis refers to government ordinance designated cities and wards of Tokyo, and medium sized cities refer to cities having a population of over 150 thousand inhabitants. Small sized cities refer to cities with a population of less than 150 thousand [12]. In addition, data on population for each municipality were obtained from the survey on population, demographics, and number of households based on the basis resident register [13], and data on population of specific age in 2015 were obtained from the census [14].

Moreover, data on air pollutant concentrations were obtained from the environmental database governed by the National Institute for Environmental Studies in Japan [15]. The database collects data on air pollutant concentrations collected from nationwide air pollution monitoring stations, and the data from 2006 to 2018 was made available to the public. In addition, the data on annual average values of sulfur dioxide $\left(\mathrm{SO}_{2}\right)$, Nitrogen dioxide $\left(\mathrm{NO}_{2}\right)$, suspended particulate matter $(\mathrm{SPM})$, carbon monoxide (CO), and photochemical oxidant (Ox) in each station were used in this study because these pollutants are known to be associated with the incidence of asthma $[7,16]$. available data.

An approval by an institutional review board was not required for this study because we used only publicly

\section{Statistical analysis}

We calculated the age-standardized prevalence of asthma for each year, gender, and regional urbanization level using the population aged 6-17 years of Japan in 2015 as standard population. We also calculated the annual percent change (APC) of the age-standardized prevalence and its 95\% confidence interval (CI) by applying a linear regression model on logarithm of the age-standardized prevalence using year as an explanatory variable.

Furthermore, we calculated the slope index of inequality (SII) and relative index of inequality (RII) for evaluating the disparity in the age-standardized prevalence of asthma with respect to regional urbanization levels $[17,18]$. These are indexes of inequality and often used in social epidemiology. The SII can be interpreted as the difference in health outcome between the lowest and the highest social ranks, and the RII can be interpreted as the ratio between the two ranks. A more detailed explanation of the indexes is available in a previous study [19]. Populations for each regional urbanization levels are necessary for calculating the indexes, and they were obtained by aggregating the population in each municipality by regional urbanization level. We obtained the $95 \% \mathrm{CI}$ of the RII by the bootstrap method. We also calculated the annual change (AC) of the SII and RII and it 95\% CI by applying a linear regression model on these indexes using year as an explanatory variable. AC represents the index value change in a year and corresponds to the regression coefficient of the linear regression model.

We aggregated the air pollutant data of each station for each municipality and calculated the mean of the annual average concentration by regional urbanization level for each year and each the air pollutant. All the statistical analysis was conducted by R3.6.3 (https://www.r-project.org/).

\section{Results}

Table 1 shows the age-standardized prevalence of asthma (\%) by year, gender, and urbanization level and its APC. The age-standardized prevalence was highest in the metropolis in 2006 for males and females. However, it significantly decreased in the study period in the metropolis for males and females, and the degree of the decrease was largest in the metropolis. Conversely, the age-standardized prevalence increased in towns and villages, and APC was greater than zero. 
Okui et al. I Trend in asthma prevalence

Table 1. Age-standardized asthma prevalence (\%) by year, gender, and regional urbanization level and its APC.

\begin{tabular}{|c|c|c|c|c|}
\hline \multirow[b]{2}{*}{ Gender and year } & \multicolumn{4}{|c|}{ Urbanization level } \\
\hline & Metropolis & $\begin{array}{c}\text { Medium sized } \\
\text { cities }\end{array}$ & Small sized cities & $\begin{array}{c}\text { Towns and } \\
\text { villages }\end{array}$ \\
\hline \multicolumn{5}{|l|}{ Males } \\
\hline 2006 & 4.74 & 3.36 & 3.16 & 3.03 \\
\hline 2007 & 4.80 & 3.31 & 3.34 & 3.54 \\
\hline 2008 & 4.73 & 3.53 & 3.29 & 3.20 \\
\hline 2009 & 4.78 & 3.40 & 3.47 & 3.32 \\
\hline 2010 & 4.85 & 3.62 & 3.47 & 3.32 \\
\hline 2011 & 4.48 & 3.85 & 3.54 & 3.86 \\
\hline 2012 & 4.68 & 3.85 & 3.66 & 3.40 \\
\hline 2013 & 4.55 & 3.71 & 3.86 & 3.61 \\
\hline 2014 & 4.10 & 3.61 & 3.50 & 3.48 \\
\hline 2015 & 4.11 & 3.56 & 3.78 & 3.60 \\
\hline 2016 & 3.90 & 3.24 & 3.54 & 3.68 \\
\hline 2017 & 3.98 & 3.41 & 3.46 & 3.90 \\
\hline 2018 & 3.62 & 3.27 & 3.24 & 3.42 \\
\hline 2019 & 3.38 & 3.10 & 3.18 & 3.28 \\
\hline $\mathrm{APC}(95 \% \mathrm{CI})$ & $-2.51(-3.19,-1.83)$ & $-0.46(-1.38,0.47)$ & $0.17(-0.74,1.08)$ & $0.72(-0.24,1.69)$ \\
\hline \multicolumn{5}{|l|}{ Females } \\
\hline 2006 & 3.10 & 2.28 & 2.11 & 2.20 \\
\hline 2007 & 3.37 & 2.19 & 2.28 & 2.51 \\
\hline 2008 & 3.21 & 2.34 & 2.16 & 2.20 \\
\hline 2009 & 3.20 & 2.26 & 2.34 & 2.26 \\
\hline 2010 & 3.21 & 2.42 & 2.45 & 2.44 \\
\hline 2011 & 2.98 & 2.64 & 2.42 & 2.69 \\
\hline 2012 & 3.12 & 2.48 & 2.43 & 2.09 \\
\hline 2013 & 3.01 & 2.45 & 2.53 & 2.43 \\
\hline 2014 & 2.85 & 2.41 & 2.36 & 2.39 \\
\hline 2015 & 2.69 & 2.39 & 2.48 & 2.49 \\
\hline 2016 & 2.70 & 2.28 & 2.45 & 2.64 \\
\hline 2017 & 2.61 & 2.31 & 2.41 & 2.71 \\
\hline 2018 & 2.49 & 2.32 & 2.12 & 2.35 \\
\hline 2019 & 2.33 & 2.29 & 2.13 & 2.22 \\
\hline $\mathrm{APC}(95 \% \mathrm{CI})$ & $-2.41(-3.00,-1.82)$ & $0.06(-0.65,0.77)$ & $0.15(-0.80,1.11)$ & $0.49(-0.69,1.69)$ \\
\hline
\end{tabular}

$\overline{\mathrm{APC}}$, annual percent change; $\mathrm{CI}$, confidence interval 
Table 2. SII and RII for age-standardized asthma prevalence (\%) depending on regional urbanization level by gender and its annual change.

\begin{tabular}{|c|c|c|c|c|}
\hline \multirow{2}{*}{ Year } & \multicolumn{2}{|c|}{ Males } & \multicolumn{2}{|c|}{ Females } \\
\hline & SII $(95 \% \text { CI })^{1}$ & RII $(95 \% \text { CI })^{2}$ & SII $(95 \% \text { CI })^{1}$ & $\operatorname{RII}(95 \% \mathrm{CI})^{2}$ \\
\hline 2006 & $1.93(-1.12,4.97)$ & $1.71(1.04,1.96)$ & $1.06(-1.02,3.14)$ & $1.54(0.94,1.82)$ \\
\hline 2007 & $1.40(-2.84,5.64)$ & $1.45(0.90,1.83)$ & $0.93(-2.62,4.49)$ & $1.43(0.80,2.06)$ \\
\hline 2008 & $1.76(-0.89,4.41)$ & $1.60(1.03,1.82)$ & $1.19(-0.93,3.30)$ & $1.61(0.97,1.86)$ \\
\hline 2009 & $1.57(-1.80,4.94)$ & $1.51(0.98,1.75)$ & $1.00(-1.36,3.37)$ & $1.48(0.95,1.73)$ \\
\hline 2010 & $1.73(-0.96,4.42)$ & $1.57(1.04,1.73)$ & $0.84(-1.21,2.89)$ & $1.37(0.98,1.59)$ \\
\hline 2011 & $0.83(-1.31,2.97)$ & $1.23(0.88,1.41)$ & $0.43(-1.03,1.89)$ & $1.17(0.85,1.40)$ \\
\hline 2012 & $1.47(-0.01,2.95)$ & $1.45(1.06,1.59)$ & $1.12(-0.12,2.37)$ & $1.55(1.02,1.77)$ \\
\hline 2013 & $0.98(-1.12,3.08)$ & $1.28(0.94,1.41)$ & $0.61(-0.77,2.00)$ & $1.26(0.96,1.39)$ \\
\hline 2014 & $0.73(-0.34,1.81)$ & $1.22(1.01,1.29)$ & $0.54(-0.56,1.63)$ & $1.24(0.98,1.36)$ \\
\hline 2015 & $0.48(-1.11,2.07)$ & $1.13(0.91,1.24)$ & $0.19(-0.73,1.12)$ & $1.08(0.94,1.16)$ \\
\hline 2016 & $0.16(-2.15,2.47)$ & $1.05(0.82,1.26)$ & $0.04(-1.57,1.65)$ & $1.02(0.80,1.23)$ \\
\hline 2017 & $0.13(-2.34,2.60)$ & $1.04(0.81,1.28)$ & $-0.11(-1.62,1.41)$ & $0.96(0.78,1.16)$ \\
\hline 2018 & $0.26(-0.99,1.52)$ & $1.08(0.92,1.20)$ & $0.25(-0.78,1.29)$ & $1.11(0.85,1.26)$ \\
\hline 2019 & $0.11(-0.87,1.08)$ & $1.03(0.92,1.12)$ & $0.20(-0.25,0.64)$ & $1.09(0.94,1.16)$ \\
\hline $\mathrm{AC}(95 \% \mathrm{CI})$ & $-0.15(-0.19,-0.11)$ & $-0.05(-0.06,-0.04)$ & $-0.09(-0.12,-0.06)$ & $-0.04(-0.06,-0.03)$ \\
\hline
\end{tabular}

$\mathrm{AC}$, annual change; $\mathrm{CI}$, confidence interval; SII, slope index of inequality; RII, relative index of inequality

${ }^{1}$ The SII can be interpreted as the difference in the age-standardized prevalence between the least urbanized area and the most urbanized area

${ }^{2}$ The RII can be interpreted as the ratio of the age-standardized prevalence between the least urbanized area and the most urbanized area

Table 2 shows the SII and RII for the age-standardized prevalence of asthma (\%) with respect to the urbanization level by gender and its AC. Values of SII $>0$ and RII $>1$ indicate that the prevalence of asthma increases with an increase in urbanization levels. However, both the SII and RII showed significant decreasing trends across the study period, and the disparity with respect to the urbanization level shrank over the years. The RII was significantly $>1$ for males and females during some years until 2014, whereas no significant disparity was observed later.

Figure 1 shows the early mean of annual average concentrations of $\mathrm{SO}_{2}, \mathrm{NO}_{2}, \mathrm{SPM}, \mathrm{CO}$, and Ox by urbanization level. The concentrations of all the air pollutants showed decreasing trends across the study period except for Ox. The concentrations of pollutants in metropolis were the highest throughout the years except for Ox, and those for towns and villages tended to be the lowest. Conversely, the degree of the decrease was largest in the metropolis for $\mathrm{NO}_{2}, \mathrm{SPM}$, and CO, and the difference in concentrations decreased between the metropolis and the other areas particularly for $\mathrm{NO}_{2}, \mathrm{SPM}_{\text {, and }}$ CO. Conversely, the concentration of Ox was high in small sized cities, towns, and villages, and the difference in concentrations decreased over the years based on the urbanization levels. 

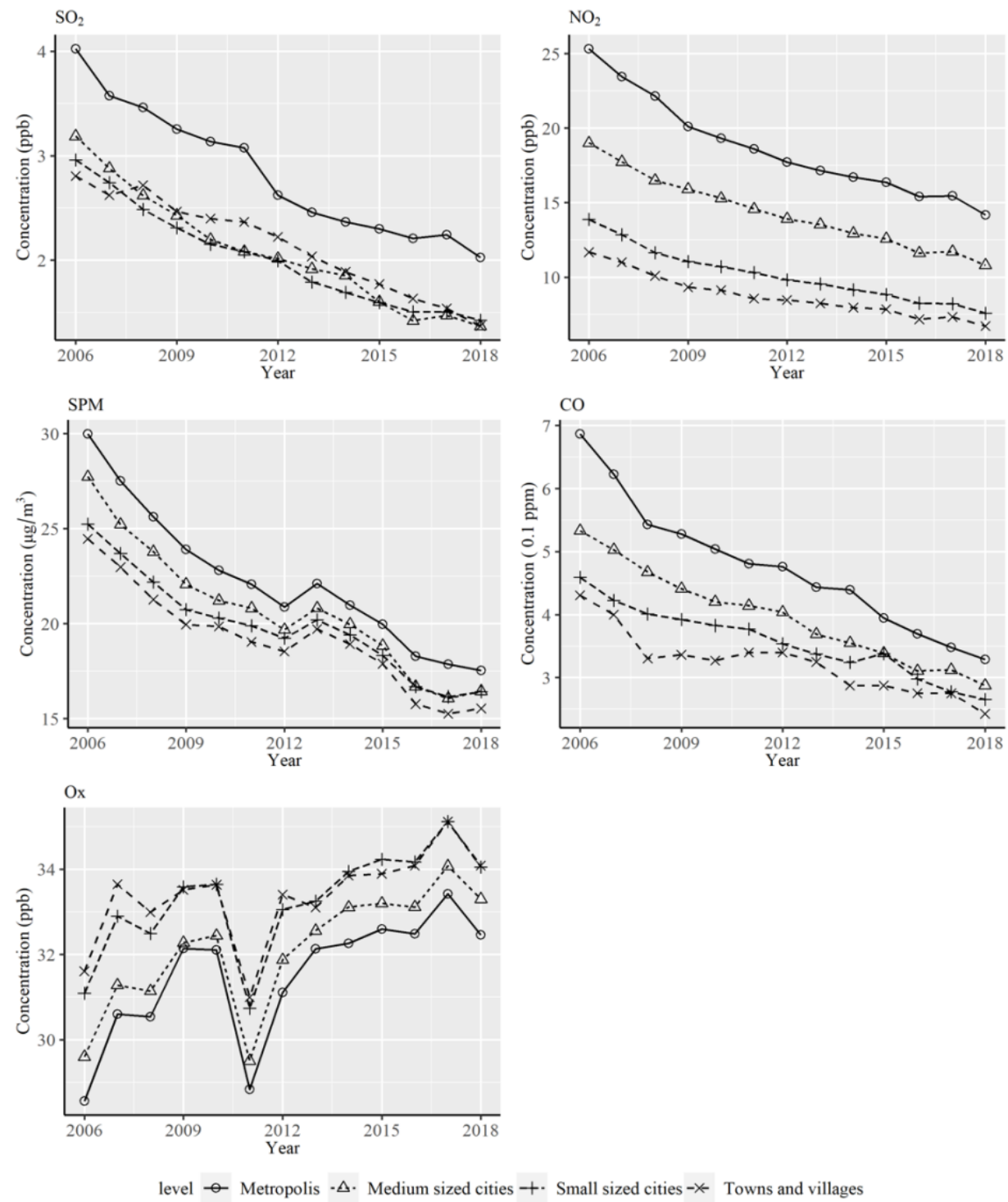

Figure 1. Yearly mean of annual average concentrations of $\mathrm{SO}_{2}, \mathrm{NO}_{2}, \mathrm{SPM}, \mathrm{CO}$, and Ox by regional urbanization level.

\section{Discussion}

In this study, we evaluated the degree of disparity in the prevalence of asthma with respect to urbanization level using RII and SII as disparity indexes and showed the changes in RII and SII values over the study period. Urbanization level-related disparity in asthma prevalence existed in Japan because SII was significantly higher than zero and RII was significantly higher than one in some of the years in the study period. In other words, the age-standardized prevalence asthma prevalence tended to increase with an increase in regional urbanization level in some years. However, AC of the RII and SII values over the years was significantly decreased, and it was shown that the disparity in the prevalence of asthma with respect to the areal urbanization level decreased over the analyzed periods for males and females. Major reasons for these results were that the age-standardized prevalence in the metropolitan areas particularly decreased across the study period, whereas those for rural areas increased. We hereby discuss possible reasons for this phenomenon.

Air pollutant concentrations in the metropolis were the highest for all pollutants in 2006 except for Ox. Air pollution problems were particularly serious in urban areas [20], and this is considered to be related to the high prevalence of asthma in 2006 like other countries [21]. Conversely, some regulations against air pollution have been implemented particularly in metropolitan areas in Japan [9], and it was shown in the present study that concentrations of $\mathrm{SPM}_{\mathrm{N}} \mathrm{NO}_{2}$, and CO particularly decreased in the metropolis in the study period. Therefore, a decrease in the prevalence of asthma in the metropolis in recent years might be caused due to a significant decrease of air pollutant concentrations. Contrarily, reasons for an increasing trend in the prevalence of asthma in towns and villages are unknown because most of the air pollutant concentrations also decreased in these areas in the study period. Concentration of Ox showed an increasing trend in the periods, and it might be related to the increasing trend for towns and villages. Or, causes other than air pollution are 
considered to be related to the trend in towns and villages. Moreover, the concentration of Ox suddenly decreased in 2011 regardless of the regional urbanization level; the reasons for this phenomenon have not been unveiled in Japan.

Smoking is another major risk factor for asthma. Passive smoking has an effect on children, and maternal smoking during pregnancy was also shown to be related to the incidence of asthma in Japan [22,23]. In Japan, the prevalence of smoking is known to be higher in metropolitan areas compared with the other areas for females [24]. The prevalence of smoking significantly decreased from 2004 to 2019, and the degree of decrease of the prevalence was a little bit larger in metropolitan areas compared with the other areas [24]. Although the degree of decrease in rural areas has not been investigated, there is a possibility that the degree of decrease of the prevalence of smoking is low in rural areas in the study period. It is known that socioeconomic differences in the smoking prevalence increased from 2001 to 2016 in Japan [25], and regional socioeconomic status might be associated with trends of smoking.

Other indoor environmental factors, such as mold and dampness, have been identified as risk factors of asthma [26]. According to a review, children in inner-city are exposed to various indoor allergens, such as mice, house dust mites, and mold, and these factors are related to the development of asthma [27]. In addition, according to a study investigating the association between indoor environmental factors and the development of asthma in Japan, reinforced concrete houses or apartment buildings increase the load of indoor air pollutants because of airtightness [28]. Therefore, differences in living environments, including indoor environmental factors, due to urbanization levels might be related to the trend and difference in asthma prevalence.

As an implication, it was suggested from the results that the prevalence of asthma might tend to be larger in towns and villages compared with the urban areas in the future if this trend continues. It was shown that the age-standardized prevalence in towns and villages showed an increasing trend in both males and females, and it is important to identify the causes of this phenomenon. Factors, such as the prevalence of smoking and obesity are possibly related to the trends because obesity is also a risk factor for asthma [29], and it is meaningful to confirm differences in trends in these factors with respect to different regions in the future. Moreover, although we focused on urbanization level, studies on other regional characteristics, such as industrial areas and non-industrial areas, will be helpful.

There are some limitations in this study. First, the School Health Statistics Survey targets only children, thus the trends in adults are unknown. A similar study targeting adults is also warranted for verifying the difference in trends for urban and rural areas. Second, we could obtain only the data after 2006 because only those data were publicly available. Analyzing the data of longer periods will be meaningful for understanding the causes of the trends in prevalence. Third, air pollution monitoring stations do not exist in all the municipalities in Japan, and the number of stations was relatively small in towns and villages. Therefore, there may be some biases in air pollutant concentrations in towns and villages. On the other hand, the results of this study are based on a nationwide survey conducted by the ministry of education, culture, sports, science and technology in Japan, and the results represent the overall trend in Japan.

\section{Conclusions}

We investigated trends in the prevalence of asthma and air pollutant concentrations by urbanization level in Japan from 2006 to 2019. We found that the age-standardized prevalence significantly decreased in the study period in the metropolis for males and females, whereas the age-standardized prevalence increased in towns and villages. Both the SII and RII showed significant decreasing trends, and the disparity with respect to urbanization level shrank over the years. The concentrations of pollutants in the metropolis were highest throughout the years for $\mathrm{SO}_{2}, \mathrm{NO}_{2}$, $\mathrm{SPM}$, and $\mathrm{CO}$, whereas the difference in concentrations of $\mathrm{NO}_{2}, \mathrm{SPM}$, and $\mathrm{CO}$ decreased between the metropolis and other areas.

\section{Acknowledgement}

Enago has proofread the manuscript.

\section{Conflict of interest}

The authors declare no conflicts of interest associated with this manuscript.

\section{ORCID}

Tasuku Okui: 0000-0001-5098-8502

\section{References}

[1] To T, Stanojevic S, Moores G, Gershon AS, Bateman ED, Cruz AA, et al. Global asthma prevalence in adults: findings from the cross-sectional world health survey. BMC Public Health 2012;12(1):1-8. https://doi.org/10.1186/1471-2458-12$\underline{204}$ 
[2] Serebrisky D, Wiznia A. Pediatric asthma: a global epidemic. Ann Glob Health 2019;85(1):6. http://doi.org/10.5334/aogh.2416

[3] Yamauchi Y. Epidemiology of asthma: the present and near future. Nihon Naika Gakkai Zasshi 2018;107(10):2059-2066. (Japanese) https://doi.org/10.2169/naika.107.2059

[4] Lawson JA, Rennie DC, Cockcroft DW, Dyck R, Afanasieva A, Oluwole O, Afsana J. Childhood asthma, asthma severity indicators, and related conditions along an urban-rural gradient: a cross-sectional study. BMC Pulm Med 2017;17(1):1-9. https://doi.org/10.1186/s12890-016-0355-5

[5] Bröms K, Norbäck D, Eriksson M, Sundelin C, Svärdsudd K. Effect of degree of urbanisation on age and sex-specific asthma prevalence in Swedish preschool children. BMC Public Health 2009;9(1):1-11. https://doi.org/10.1186/1471-2458$\underline{9-303}$

[6] Ando Y, Aida J. Regional Differences in Health Status in Japanese School Children, by Using National Statistics. Health Science and Health Care._2007;7(2):108-114. (Japanese) https://www.fihs.org/en/volume7 2/article9.pdf

[7] Tiotiu AI, Novakova P, Nedeva D, Chong-Neto HJ, Novakova S, Steiropoulos P, et al. Impact of air pollution on asthma outcomes. Int J Environ Res Public Health 2020;17(17):6212 (Japanese). https://doi.org/10.3390/ijerph17176212

[8] Guo P, Yokoyama K, Suenaga M, Kida H. Mortality and life expectancy of Yokkaichi asthma patients, Japan: late effects of air pollution in 1960-70s. Environ Health 2008;7(1):1-10. https://doi.org/10.1186/1476-069X-7-8

[9] Yokota $\mathrm{H}$. The role that the local government played in improving the air quality (the Case of Tokyo). J Jpn Soc Atmos Environ 2020;55(3):A60-A65. (Japanese) https://doi.org/10.11298/taiki.55.A60

[10] Hara K, Homma J, Tamura K, Inoue M, Karita K, Yano E. Decreasing trends of suspended particulate matter and PM2.5 concentrations in Tokyo, 1990-2010. J Air Waste Manag Assoc 2013;63(6):737-748. https://doi.org/10.1080/10962247.2013.782372

[11] Ministry of Education, Culture, Sports, Science in Japan. The school health statistics survey. Assessed on Oct 1, 2021. https://www.e-stat.go.jp/stat-search/files?page $=1 \&$ toukei $=00400002 \&$ tstat $=000001011648$

[12] Ministry of Education, Culture, Sports, Science in Japan. Overview of the school health statistics survey. Assessed on Oct 1, 2021. https://www.mext.go.jp/b menu/toukei/chousa05/hoken/1268826.htm

[13] Ministry of Internal Affairs and Communications in Japan. The survey on population, demographics, and number of households based on the basis resident register. Assessed on Oct 1, 2021.

https://www.e-stat.go.jp/stat-search/files?page=1\&toukei=00200241\&tstat=000001039591

[14] Ministry of Internal Affairs and Communications in Japan. The census. Assessed on Oct 1, 2021. https://www.e-stat.go.jp/stat-search/files?page=1\&toukei=00200521\&result page $=1$

[15] The National Institute for Environmental Studies in Japan. The environmental database. Assessed on Oct 1, 2021. https://www.nies.go.jp/igreen/

[16] Kumar R, Nagar JK, Goel N, Kumar P, Kushwah AS, Gaur SN. Indoor air pollution and asthma in children at Delhi, India. Advances in Respiratory Medicine 2015;83(4):275-282. https://doi.org/10.5603/PiAP.2015.0047

[17] Mejia G, Jamieson LM, Ha D, Spencer AJ. Greater inequalities in dental treatment than in disease experience. J Dent Res 2014;93(10):966-971. https://doi.org/10.1177/0022034514545516

[18] Chang Y, Kang HY, Lim D, Cho HJ, Khang YH. Long-term trends in smoking prevalence and its socioeconomic inequalities in Korea, 1992-2016. Int J Equity Health 2019;18(1):1-10. https://doi.org/10.1186/s12939-019-1051-x

[19] Renard F, Devleesschauwer B, Speybroeck N, Deboosere P. Monitoring health inequalities when the socio-economic composition changes: are the slope and relative indices of inequality appropriate? Results of a simulation study. BMC Public Health 2019;19(1):1-9 https://doi.org/10.1186/s12889-019-6980-1

[20] Zhang J, Dai J, Yan L, Fu W, Yi J, Chen Y, et al. Air pollutants, climate, and the prevalence of pediatric asthma in urban areas of China. Biomed Res Int 2016;2016:8. https://doi.org/10.1155/2016/2935163 
[21] Lin RS, Sung FC, Huang SL, Gou YL, Ko YC, Gou HW, et al. Role of urbanization and air pollution in adolescent asthma: a mass screening in Taiwan. J Formos Med Assoc 2001;100(10):649-655.

[22] Furuhata M, Otsuka Y, Kaneita Y, Nakagome S, Jike M, Itani O, et al. Factors associated with the development of childhood asthma in Japan: a nationwide longitudinal study. Matern Child Health J 2020;24(7):911-922. https://doi.org/10.1007/s10995-020-02944-0

[23] Yoshida S, Mishina H, Takeuchi M, Kawakami K. Association of prenatal maternal, prenatal secondhand, and postnatal secondhand smoking exposures with the incidence of asthma/atopic dermatitis in children: An epidemiological study using checkup data of mothers and children in Kobe city. Nihon Koshu Eisei Zasshi 2021;68(10):659-668 (Japanese). https://doi.org/10.11236/jph.20-142

[24] Okui T. An age-period-cohort analysis of the difference in smoking prevalence between urban and non-urban areas in Japan (2004-2019). Epidemiol Health 2020;42:e2020072. https://doi.org/10.4178/epih.e2020072

[25] Tanaka H, Mackenbach JP, Kobayashi Y. Widening socioeconomic inequalities in smoking in Japan, 2001-2016. J Epidemiol 2021;31(6):369-377. https://doi.org/10.2188/jea.JE20200025

[26] Oluwole O, Kirychuk SP, Lawson JA, Karunanayake C, Cockcroft DW, Willson PJ, et al. Indoor mold levels and current asthma among school-aged children in Saskatchewan, Canada. Indoor Air 2017;27(2):311-319.

https://doi.org/10.1111/ina.12304

[27] Kanchongkittiphon W, Gaffin JM, Phipatanakul W. The indoor environment and inner-city childhood asthma. Asian Pac J Allergy Immunol 2014;32(2):103-110.

[28] Nakajima T, Azuma E, Hashimoto M, Toyoshima K, Hayashida M, Komachi Y. Factors aggravating bronchial asthma in urban children (I)--The involvement of indoor air pollution. Nihon Koshu Eisei Zasshi Japanese journal of public health 1998;45(5):407-422 (Japanese).

[29] Okabe Y, Adachi Y, Itazawa T, Yoshida K, Ohya Y, Odajima H, et al. Association between obesity and asthma in Japanese preschool children. Pediatr Allergy Immunol 2012;23(6):550-555.

https://doi.org/10.1111/j.1399-3038.2011.01261.x 